\title{
A New MAC Protocol PB-TDMA for WSNs Based on Event-driven
}

\author{
Hui Wang, Xuefeng Li, Yunfeng Wang \\ College of Computer Science and Technology, \\ Henan Polytechnic University \\ Jiaozuo, China \\ wanghui_jsj@hpu.edu.cn, \\ lixuefeng3710@gmail.com, \\ 786161871@qq.com
}

\begin{abstract}
In order to improve the WSN, a novel WSN protocol based on the event-driven named PB-TDMA is proposed. One of the problems in the WSN protocol is that each node's probability of data delivery is usually diverse at different time and environment. This paper puts forward a method to resolve such a problem. It makes two nodes with different probability share a time slot which could use to compress the frame length and enhance the utilization ratio of channel. In addition, through running statistics on the probability of each node's data sending, it proposes a new allocation method of time slot to avoid data collision. On the basis of the above strategy, we can constantly update the delivery rate to achieve the goal of adaptive. Simulation results show that PB-TDMA protocol performs better for wireless sensor network with high-density deployment and moderate load.
\end{abstract}

Keywords: Wireless Sensor Network, Event-driven, MAC Protocol, Energy Consumption

\section{Introduction}

In 2003 the United States, "Technology Review" magazine in his discussion of the next ten emerging technologies, ranked the WSN as the first item. Wireless sensor node as a micro-device, has limited power supply energy. So the energy consumption of the node has a direct impact on the lifetime of the node. By controlling the acquisition of Wireless media and data dissemination, the MAC protocol with excellent performance not only can reduce the energy and increase throughput, but also effectively decrease the rate of the package loss [1].

Commonly, MAC protocol is applied to optimize energy consumption and balance network performance according to different application scenarios and criteria. In terms of data delivery, WSNs can be categorized into 3 groups as periodic awareness, event-driven awareness [2], query-driven awareness, and awareness. Periodic awareness means that WSNs can acquire and send data at a defined period and enter a sleep state as soon as possible out of the defined period. .However, sensor nodes sensing the specific phenomena or measurement values defined previously should send the data immediately in event-driven ones. Hence, the application requirements are so different that application-specific designs are necessary. This paper mainly focuses on the event-driven type of WSNs.

MAC protocol nodes based on event-driven send data at uncertain time cycle, it only send data when the data exceed threshold. Compared with other kinds of protocol, MAC protocol based on event-driven is more suitable for earthquake, fire, 
accident warning of mine, target tracking etc. However, Some others, such as SMAC [3,4],T-MAC[5] always have a fixed time cycle, which may make them deliver data in its sleep state rather at the defined period and may not send data to sink node in time. Because of these features, they are not suitable for some applications. The traditional TDMA protocol also allocates separate time slot to each node sending data. Because the transmission data probability is low, the bandwidth utilization will be very low and take great waste of energy.

Energy-Efficient TDMA [6] improves the traditional TDMA protocol. It suggests that nodes which don't send date in its allocated time slot should be in the sleep state. Although the improvements can decrease the energy of ordinary nodes, cluster nodes remain idle listening state and can not increase the energy efficiency.

Liu et al. propose the E-MAC [7], a MAC protocol which is designed based on the attributes of EDDA(Event-driven data aggregation). It classifies the nodes in EDDA scenario and divides the transportation of data packets into several stages. By the ways of sequential wake-up, multi-hop reservation and interlaced transmission, E-MAC schedules and sends data packets from the event zone to sink nodes in only one time cycle. It offers its favorable performance with a low energy cost in EDDA scenario, but delay and reliability become the serious problem.

G.M. Shafiullah design the Energy-Efficient Adaptive TDMA [8] (EA-TDMA), in which cluster heads will send their time slot to all cluster nodes after clusters are established. When the time slot wakes up, every node needs to check its buffer and find out whether there are some data to be sent. The nodes should be in the sleep state rather in the state of idle monitor if there are not some data to be sent. However, it doesn't take into account the energy consumption among cluster head balance.

BMA [9] (Bit-map Assisted) stipulates that there is a contention period when each frame begins and each node is given 1 bit time slot to report the cluster head, whether it has data to send or not. A node with data transmitted called a source node, initially sends 1 bit to the cluster head. A non-source node without data transmitted leaves its own 1-bit time slot unused. Thus, the $\mathrm{CH}$ can distinguish the source nodes and can prepare and broadcast the time slot allocation schedule according to this knowledge. Finally, while non-source nodes fall directly asleep, source nodes will transmit their sensed data during their allocated time slots. However, due to the length of each frame is fixed, the channel utilization is low. Then there is a huge scheduling overhead, especially the number of nodes. Although Nukhet Sazak improves the BMA [10], it doesn't change the fixed length of frame and further aggravate the scheduling overhead.

Above protocols design some unique methods to solve problems of energy consumption, delay and channel utilization, but they have not yet considered that nodes in different geographical position and different time will have difference of transmission data probability. In this paper, we mainly consider this problem and try to design a new protocol (PB-TDMA) to solve it.

\section{Protocol Description}

The biggest characteristic of WSN based on event-driven is that the WSN doesn't send data to cluster-head in strict accordance with cycles until it satisfies that the monitoring data is more than the threshold. So the probability of node's transmission data is quite low and different each other, especially in the condition of different geographical position and time distribution. The PB-TDMA protocol designed based on this condition schedules two nodes to one time slot so that to send data reasonably. Meanwhile the disadvantage of this approach is collision. So 
we design a strategy statistics the probability of node's transmission data to earn the reasonable time slot distribution.

The main objective of the PB-TDMA protocol is to reduce the waste of energy due to idle listening and collision while maintaining good performance. The operation of PB-TDMA is divided into rounds as in LEACH [11].After completing the operation, networks will enter the initial phase which will be only done once. In the initial phase, E-TDMA protocol is used in networks, and each node records the number $\mathrm{n}$ of sending data and then calculates the probability $\eta=\frac{n}{N} \times 100 \%$ of node's transmission data through the number $\mathrm{N}$ of frame, the probability will be update in steady phase and is as the basis of time slot allocation.

Steady phase will be taken at the end of the initial phase. Cluster head determines the time slot of each node according to the probability $\eta$. Its structure is shown in Figure 1. For instance, there are 10 nodes in the cluster and the probability of the nodes' transmission data is S1 to S10.However, the whole frame just needs to set up seven time slots instead of ten in other protocols. The allocation of each time slot is shown as in Table 1. Due to the soft threshold and hard threshold setting in eventdriven MAC protocol, the probability of the nodes' transmission data is far lower than other MAC protocols, so we can use this strategy to achieve efficient use of time slot. During the later stable phases, cluster head will aggregate the probability of the nodes' transmission data. If the sorting of probability changes, cluster head will redistribute time slots in the following round; Otherwise, ordinary node will work according to the last slot allocation. In this way, we can achieve the goal of adaptive and save energy of cluster head at the same time.

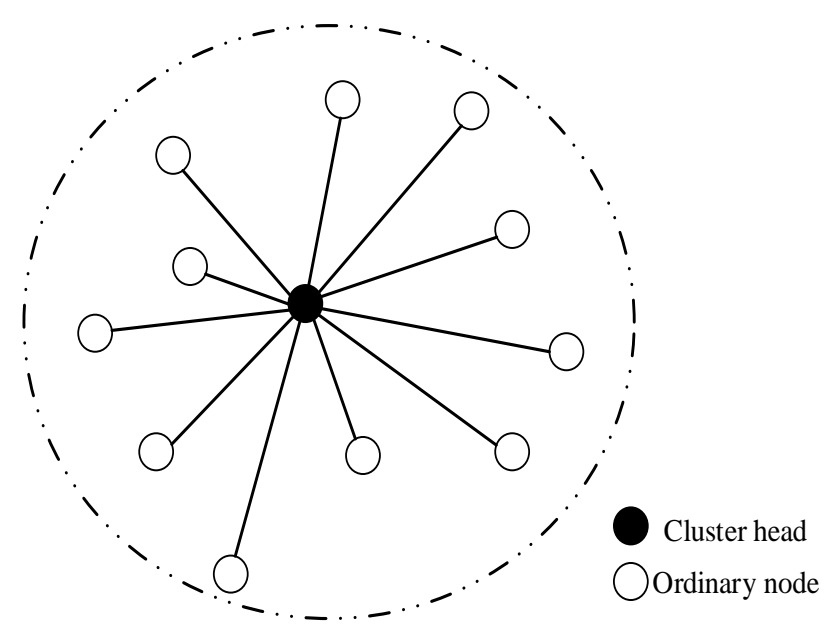

Figure 1. Cluster Structure

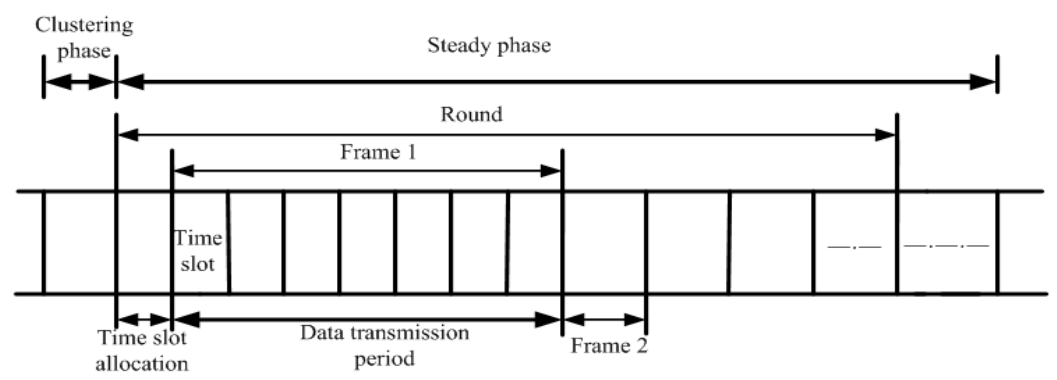

Figure 2. Network Cycle Structure 
Steady phase will be taken at the end of the initial phase. Cluster head determines the time slot of each node according to the probability $\eta$. Its structure is shown in Figure 2. For instance, there are 10 nodes in the cluster and the probability of the nodes' transmission data is $\mathrm{S} 1$ to $\mathrm{S} 10$.However, the whole frame just needs to set up seven time slots instead of ten in other protocols. The allocation of each time slot is shown as in Table 1. Due to the soft threshold and hard threshold setting in eventdriven MAC protocol, the probability of the nodes' transmission data is far lower than other MAC protocols, so we can use this strategy to achieve efficient use of time slot. During the later stable phases, cluster head will aggregate the probability of the nodes' transmission data. If the sorting of probability changes, cluster head will redistribute time slots in the following round; Otherwise, ordinary node will work according to the last slot allocation. In this way, we can achieve the goal of adaptive and save energy of cluster head at the same time.

Table 1. Time Slot Allocation

\begin{tabular}{|l|l|l|l|l|l|l|}
\hline Slot 1 & Slot 2 & Slot 3 & Slot 4 & Slot 5 & Slot 6 & Slot 7 \\
\hline S10 S9 & S6 S8 & S5 S7 & S4 S7 & S3 S8 & S2 S7 & S1 S8 \\
\hline
\end{tabular}

\section{Analysis of the Energy Consumption and the Delay}

The major sources of energy consumption are idle listening, collision, overhearing, and packet control overhead in WSNs. The radio of a sensor node can operate in four different modes: Transmit, Receive, Idle and Sleep. We can ignore the energy consumption of sleep. The energy consumption during the transmit mode, the receive mode and the idle mode are denoted by $P_{t}, P_{r}$ 和 $P_{i}$, respectively. When a source node spends $\mathrm{T}$ seconds transmitting or receiving a packet, the energy dissipated is computed as: $E_{t}=P_{t} T, E_{r}=P_{r} T$ or $E_{i}=P_{i} T$.

We let $T_{d}$ be the time required to transmit or receive a data packet and $T_{c}$ be the time required to transmit or receive a control packet.

In PB-TDMA protocol, each time slot is arranged two nodes to send data, they wake up at the beginning of time slot which are assigned to them. The difference is that it will send data directly if the first node owns data to send, , otherwise it will continue to sleep; But if the second node owns data to send, it need wait for some time and listen to whether the channel is busy or not. If the channel is busy, the node will sleep immediately, else it will send data to cluster head. Meanwhile networks can add following strategy based on the requirements of delay. If S8 has sent data in the third time slot, it will not wake up in next few time slots, even though it has data to send. In addition, S9 and S10 reverse their order every frame, it avoids a node to not be able to send data in extreme cases.

By means of these strategies, we suppose that it has ten ordinary nodes in cluster. For PB-TDMA, the energy consumption can be computed as follows:

The energy dissipated in a source node is computed as:

$$
E_{s n}=P_{t} T_{d}
$$

A non-source node will not wake up, the energy can be computed as: 


$$
E_{\text {in }}=0
$$

The energy consumed by the slot allocation can be expressed as:

$$
E_{s c}=10 P_{r} T_{h}+P_{t} T_{h}
$$

The energy consumption of cluster head is:

$$
E_{c h}=n_{i} P_{r} T_{d}+\left(8-n_{i}\right) P_{i} T_{d}
$$

Hence, the system energy dissipated during the $i$ th frame is :

$$
E_{s i}=n_{i} E_{s n}+E_{c h}=n_{i}\left(\mathrm{P}_{t} T_{d}+\mathrm{P}_{r} T_{d}\right)+\left(8-n_{i}\right) \mathrm{P}_{i} T_{d}
$$

The total system energy expended during each round can be computed as follows

$$
E_{\text {round }}=E_{s c}+\sum_{i=1}^{k} E_{s i}=10 \mathrm{P}_{r} T_{h}+\mathrm{P}_{t} T_{h}+k n_{i}\left(\mathrm{P}_{t} T_{d}+\mathrm{P}_{r} T_{d}\right)+\left(8-n_{i}\right) \mathrm{P}_{i} T_{d}
$$

The average packet latency can be shown as :

(7)

$$
L=\frac{T_{h}}{k}+\frac{8 T_{d}}{n_{i}}
$$

$n_{i}$ is the number of source node; $\mathrm{k}$ is the number of frame in a round.

In order to explore the energy consumption of the PB-TDMA protocol and analyze its advantages and disadvantages, we compare it with BMA protocol. We add $T_{h}$ to be the time required to transmit a control frame.

In steady phase, the energy consumption of the BMA as follows:

Source nodes: $E_{\mathrm{sn}}=P_{t} T_{c}+9 P_{i} T_{c}+P_{r} T_{h}+P_{t} T_{d}$

Non-source node: $E_{\text {in }}=P_{r} T_{h}+10 P_{i} T_{c}$

Cluster head : $E_{c h}=n_{i}\left(P_{r} T_{c}+P_{r} T_{d}\right)+\left(10-n_{i}\right) P_{i} T_{c}+P_{t} T_{h}$

The energy dissipated during the $i$ th frame is :

$E_{s i}=n_{i} E_{s n}+\left(10-n_{i}\right) E_{i n}+E_{c h}=n_{i} P_{t} T_{c}+10 P_{r} T_{h}+n_{i} P_{t} T_{d}+n_{i} P_{r} T_{c}+n_{i} P_{r} T_{d}+P_{t} T_{h}+\left(110-2 n_{i}\right) P_{i} T_{c}$

The total system energy expended during each round can be computed as :

$$
E_{\text {round }}=\sum_{i=1}^{k} E_{s i}=n_{i} k P_{t} T_{c}+10 k P_{r} T_{h}+k n_{i} P_{t} T_{d}+k n_{i} P_{r} T_{c}+k n_{i} P_{r} T_{d}+k P_{t} T_{h}+\left(110-2 n_{i}\right) \mathrm{k} P_{i} T_{c}
$$

(9)

The average packet latency can be shown to be :

$$
L=\frac{10 T_{C}+T_{h}+n_{i} T_{d}}{n_{i}}
$$

As you can see from the data, PB-TDMA increases the idle listening time of cluster head, but there is no contention period and greatly reduces energy consumption of non-source nodes; Time slot becoming from allocating once a week 
to none once greatly reduces the scheduling overhead. So we get a conclusion that the energy efficiency is better than BMA and the length of frame is much shorter than BMA and channel capacity increases effectively due to a slot time assigning two nodes.

\section{Experimental Evaluation}

In order to intuitively analyze the protocol performance, we compare PB-TDMA protocol with the BMA, E-TDMA and conventional TDMA protocol in terms of energy dissipation. In the simulation experiment, the transmitted power is $462 \mathrm{~mW}$, the received power is $346 \mathrm{~mW}$, the idle listening is $330 \mathrm{~mW}$, the data rate is $24 \mathrm{kbps}$ and the size of a data packet is 300 bytes and the size of a control packet is 20 bytes.

Figure 3 is drawn by noting the above conditions to compare the channel utilization rate of the E-TDMA, TDMA, BMA and PB-TDMA under different load. In Fig. 3 the number of $\mathrm{N}=10$ and $\mathrm{K}=4$ and the channel utilization of TDMA and $\mathrm{E}-$ TDMA is quite low in low-load. Because two protocols use the fixed time slot. BMA sends slot scheduling package after collecting the number of source nodes, so the channel utilization is better than TDMA and E-TDMA. But the fixed length of frame limits the improvement of channel utilization. By assigning reasonable time slot, the PB-TDMA has a high channel utilization and the frame length is compressed.

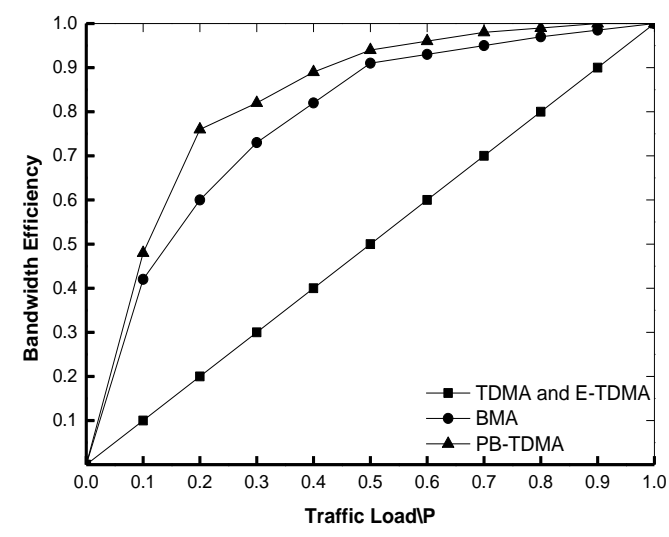

Figure 3. Channel Utilization Analysis Under Different Load

In Figure 4, we compare MAC schemes in terms of energy, where nodes are variables and probability $\mathrm{p}$ range from 0.2 to 0.5 . It shows that the PB-TDMA protocol outperforms BMA, TDMA and E-TDMA protocols in terms of energy consumption. The PB-TDMA protocol will save more energy if the number of nodes increases in the network. Hence, the EA-TDMA is suitable for large networks. 


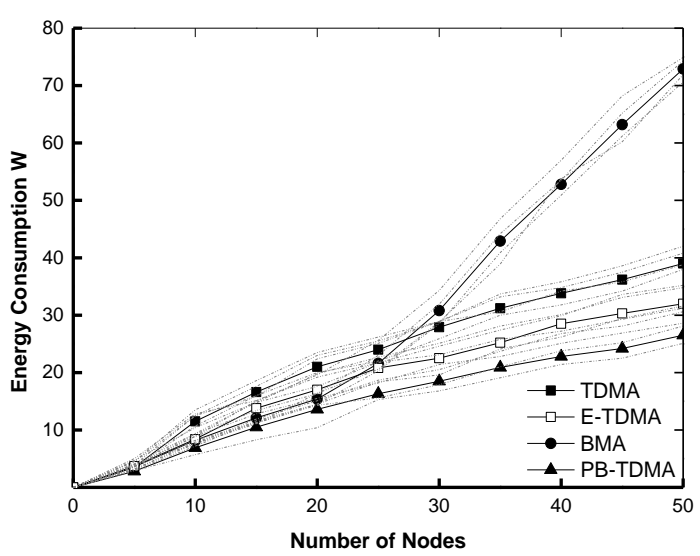

Figure 4. Energy Consumption Analysis under Different Number

From Figure 5, it is observed that the PB-TDMA protocol will save more energy if the load is different and outperforms BMA, E-TDMA and TDMA protocol in terms of energy dissipation.

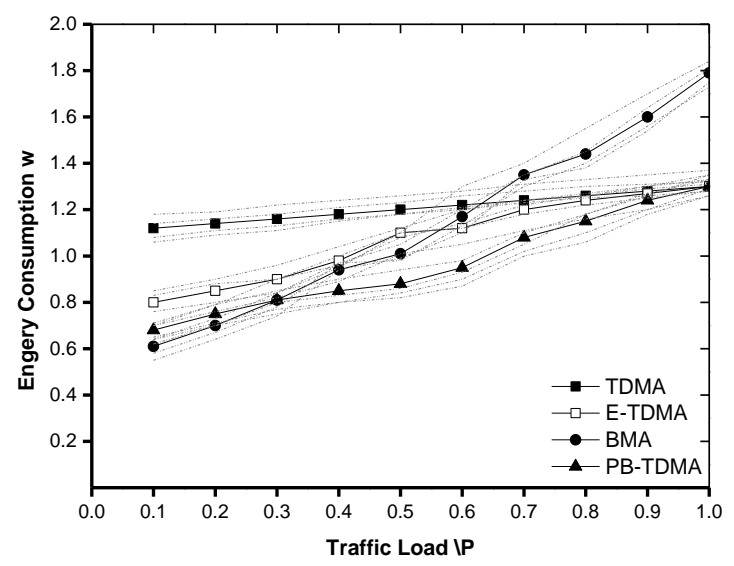

Figure 5. Energy Consumption Analysis under Different Load of Nodes

\section{Conclusion}

In this paper, we propose the PB-TDMA protocol to improve the WSN by considering the probability distribution of data. A model is developed for the proposed PB-TDMA protocol and is used to compare its performance with conventional TDMA, E-TDMA and BMA protocol. The simulation experiment shows that PB-TDMA protocol is superior to conventional TDMA, E-TDMA and BMA in most cases, especially when the probability $\mathrm{p}$ of distribution of data ranges from 0.3 and 0.6. With the condition of the same cycle, the PB-TDMA consumes less energy. So the PB-TDMA can be applied to high node density and moderate load.

\section{Acknowledgements}

This project is supported by the National Natural Science Foundation of China (No. 51174263), supported by Research Fund for the Doctoral Program of Higher 
Education of China (No. 20124116120004), and supported by Educational Commission of Henan Province of China (No. 13A510325)

\title{
References
}

[1] P. Huang, L Xiao and S. Soltani. The evolution of MAC protocols in wireless sensor networks: A survey J. Communications Surveys \& Tutorials, IEEE. 1, 15 (2013).

[2] I. Demirkol, C. Ersoy and F. Alagoz. MAC protocols for wireless sensor networks: a survey J. Communications Magazine, IEEE. 4, 44 (2006).

[3] Ye W, Heidemann J, Estrin D, "An Energy-Efficient MAC protocol for Wireless Sensor Networks," in Proc. IEEE INFOCOM, (3), (2002), pp. 1567-1576.

[4] W. Ye, J. Heidemann, D. Estrin, "Medium Access Control with Coordinated Sleeping for Wireless Sensor Networks" [J]. IEEE/ACM Transation on Networking, vol .12, no. 3, (2004) pp. 493-506.

[5] T V Dam, K Langendoen. "An Adaptive Energy Efficient MAC Protocol for Wireless Sensor Networks",[C].in Proc.of 1st ACM Conference on Embedded Nerworked Sensor Systems(SenSys'03), Los Angeles,CA, no. 11, (2003) pp. 171-180..

[6] L. Jing, Y. Georgios. Lazarou: "Modeling the energy consumption of MAC schemes in wireless clusterbased sensor networks". Proceedings of the 15th IASTED International Conference on Modeling and Simulation. Marina del Rey, California, USA, March, (2004), pp. 313-318.

[7] G. Liu, G. Yao. "E-MAC: An event-driven data aggregation MAC protocol for wireless sensor networks", Consumer Communications and Networking Conference (CCNC), (2012):pp. 941-946.

[8] G. M. Shafiullah, A. Thompson and J. Peter. Wolfs, and Shawkat Ali: "Energy-efficient TDMA MAC protocol for wireless sensor networks applications". Proceedings of International Workshop on Internet and Distributed Computing, Khulna, Bangladesh, (2008), pp. 85-90.

[9] L. Jing, Y. Georgios. Lazarou: "A bit-map assisted energy-efficient MAC scheme for wireless sensor networks". Proceedings of 3rd International Symposium on Information Processing in Sensor Networks, Berkeley, California, USA, (2004), pp. 55-60.

[10] N. Sazak, İ. Erturk and E. Koklukaya. "An event-driven WSN MAC protocol design based on active node and dynamic time slot allocation", J . Turkish Journal of Electrical Engineering \& Computer Sciences. 3, 21, (2013).

[11] W. R. Heinzelman, A. P. Chandrakasan and H. Balakrishnan. An Application-Specific Protocol Architecture for Wireless Microsensor Networks J. IEEE On Wireless Communications Trans. 4, 1, (2002).

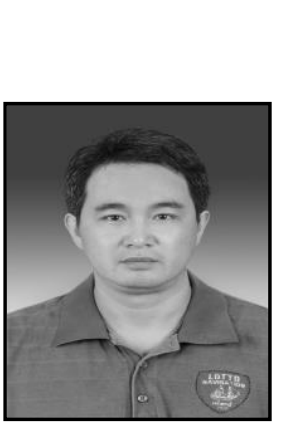

\begin{abstract}
Authors
Hui Wang, he received the Ph.D. Degree in Computer Science and Technology (2009) from Jilin University. Currently he is an Associate Professor at Henan Polytechnic University, China. His research interest is in Network \& Information Security.
\end{abstract}

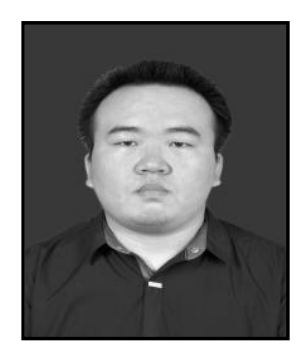

Xuefeng Li, he is a postgraduate student at Henan Polytechnic University, China. His research interest is in Network \& Information Security.

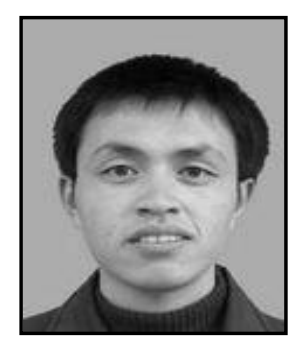

Yunfeng Wang, he is a postgraduate student at Henan Polytechnic University, China. His research interest is in Network \& Information Security. 